\title{
SOURCE SOLUTIONS FOR THE NONLINEAR DIFFUSION-CONVECTION EQUATION
}

\author{
G. C. SANDER ${ }^{1}$, R. D. BRADDOCK ${ }^{2}$, I. F. CUNNING ${ }^{2}$, J. NORBURY ${ }^{3}$ and \\ S. W. WEEKS ${ }^{1}$
}

(Received 28 March 1995; revised 27 September 1995)

\begin{abstract}
In the paper King [8], a new class of source solutions was derived for the nonlinear diffusion equation for diffusivities of the form $D(c)=D_{0} c^{m} /(1-v c)^{m+2}$. Here we extend this method for the nonlinear diffusion and convection equation

$$
\frac{\partial c}{\partial t}=\frac{\partial}{\partial z}\left[D(c) \frac{\partial c}{\partial z}-K(c)\right],
$$

to obtain mass-conserving source solutions for a nonlinear conductivity function $K(c)=$ $K_{0} c^{m+2} /(1-v c)^{m+1}$. In particular we consider the cases $m=-1,0$, and 1 , where fully analytical solutions are available. Furthermore we provide source solutions for the exponential forms of the diffusivity and conductivity as given by $D(c)=D_{0} c^{-2} e^{-n / c}$ and $K(c)=K_{0} c e^{-n / c}$.
\end{abstract}

\section{Introduction}

A variety of transport processes can be described by the one-dimensional conservation law

$$
\frac{\partial c}{\partial t}=-\frac{\partial F}{\partial z}
$$

where $c(c \geq 0)$ and $F$ are the mass and flux densities respectively, $t$ is time and $z$ is a spatial variable. Consider the specific form of $F$ given by

$$
F=-D(c) \frac{\partial c}{\partial z}+K(c)
$$

\footnotetext{
'Faculty of Science and Technology Griffith University, Nathan 4111, Australia

${ }^{2}$ Faculty of Environmental Sciences Griffith University, Nathan 4111, Australia

${ }^{3}$ Mathematical Institute, Oxford University 24-29 St Giles, Oxford, OX1 3LB

(C) Australian Mathematical Society, 1997, Serial-fee code 0334-2700/97
} 
where $D(c)$ is a concentration-dependent diffusivity and $K(c)$ a concentration-dependent convective velocity. This form of $F$ gives rise to the diffusion-convection equation which, in the past, has been applied to many areas; for example, porous media flow [12], dopant diffusion in semi-conductors [8] and the movement of a thin liquid film under gravity [5]. For applications to porous media flow in soils, the convective term $K(c)$, known as the conductivity, arises due to the influence of gravity on the flow. The soil surface is usually taken as $z=0$ with $z>0$ being in the downward direction. A consideration of flow in the upward direction can be obtained from (1.1) by replacing $K(c)$ with $-K(c)$ so that $z=0$ could then represent either the position of the water table or an impervious layer. Thus solutions will be given for $K(c)$ both positive and negative to represent flow in either direction of interest.

In this paper, we consider the conservative redistribution (by diffusion and convection) of an initial quantity of material $Q(0<Q<\infty)$ in the semi-infinite half-plane $z>0$, with a zero flux condition at the origin. This problem is described by (1.1), together with the boundary conditions

$$
\begin{gathered}
-D(c) \frac{\partial c}{\partial z}+K(c)=0, \quad z=0, \\
c \rightarrow 0, \quad z \rightarrow \infty,
\end{gathered}
$$

along with the conservation requirement

$$
Q=\int_{0}^{\infty} c(z, t) d z
$$

Obviously a realistic mathematical description of redistribution needs to include the effects of hysteresis in both $D(c)$ and $K(c)$. However this is presently available only through numerical methods as demonstrated by Watson and Sardana [20]. If we were to consider the example of nonhysteretic redistribution of water applied at the soil surface where the boundary wetting curve of the hysteresis loop was used to determine $D$ and $K$, then the effect of ignoring hysteresis is to allow water to drain from the soil surface too quickly. Thus the primary effect of hysteresis in practice is to retard redistribution and to retain water for greater time periods near the soil surface.

It has been found that soils studied in the laboratory tend to have larger hysteretic loops than field soils (Watson et al. [19] ) and that coarse grained soils as opposed to fine grained soils have marked hysteresis. Therefore the usefulness and applicability of the new solutions presented here will come from modelling the redistribution of water in fine grained field soils. Additional nonhysteretic redistribution solutions can be found for finite domains in Sander et al. [16] , and semi-infinite domains in Warrick et al. [18] and Broadbridge and Rogers [1], all of which consider arbitrary initial conditions. 
It is well known that the diffusion equation (with $K=0$ in (1.1)) admits similarity solutions for arbitrary $D(c)$ and analytic solutions are available for some classes of functional forms of $D(c)$; for example, see [6] and [8]. In [8] a new set of exact source solutions were constructed for $D(c)=D_{0} c^{m} /(1-v c)^{m+2}$ and $D(c)=$ $D_{0} c^{-2} \exp (-n / c)$ with $\nu$ an arbitrary parameter and $n, D_{0}$ positive, by inverting the previously known solutions for $D(c)=D_{0} c^{m}$ and $D_{0} e^{-n c}$ through a reciprocal Bäcklund transformation.

Lisle and Parlange [10] have conducted a symmetry analysis of (1.1) with $K=0$ and arbitrary $D$. They found that under the Boltzmann variable $c=f(\xi), \xi=x / t^{1 / 2}$, the resulting ordinary differential equation admits point symmetries if and only if $D(c)$ is of the form

$$
D(c)=\frac{1}{a_{2} c^{2}+a_{1} c+a_{0}} \exp \int \frac{b d c}{a_{2} c^{2}+a_{1} c+a_{0}},
$$

for $a_{0}, a_{1}, a_{2}$ and $b$ constants with at least one of $a_{0}, a_{1}, a_{2}$ positive. They show that the power law, exponential and Fujita [4] diffusivities are just special cases of (1.4). The diffusivities of King [8] are also simplifications of (1.4).

Hogarth et al. [7] have shown that (1.1) and (1.2) admit similarity solutions when both $D(c)$ and $K(c)$ have a general power law dependence on $c$. For simple linear and quadratic power law functions for $D$ and $K$, the resulting ordinary differential equation of [7] can be integrated to obtain closed-form solutions ([13]). In two independent studies by Edwards [2] and Yung et al. [21] a full group classification of (1.1) was performed to determine all functions $D$ and $K$ for which (1.1) admits symmetries. The similarity solutions of Hogarth et al. [7] are of course included in their analysis. Interestingly, while all possible diffusivities found by [2] and [21] were included in (1.4), there does not appear to be a comparable single encompassing formula for all the corresponding conductivities.

In this paper, we apply King's method to the symmetry reductions of [2] in order to generate a new class of highly nonlinear source solutions which include the effects of both diffusion and convection. In particular we only concentrate on those cases of $D$ and $K$ for which the reduced ordinary differential equation presented in Table 3 of Edwards [2] are straightforward to integrate. These cases were predominantly covered in Philip [13].

\section{Bäcklund transformation for the diffusion-convection equation}

Following [9], the conservation equation (1.1) is transformed to the reciprocally associated conservation equation

$$
\frac{\partial c^{\prime}}{\partial t^{\prime}}=\frac{\partial}{\partial x}\left[D^{\prime}\left(c^{\prime}\right) \frac{\partial c^{\prime}}{\partial x}-K^{\prime}\left(c^{\prime}\right)\right]
$$


by using the reciprocal Bäcklund transformation

$$
\begin{aligned}
\frac{\partial c^{\prime}}{\partial x} & =\frac{v}{(1-v c)^{3}} \frac{\partial c}{\partial z} \\
\frac{\partial c^{\prime}}{\partial t^{\prime}} & =\frac{v}{(1-v c)^{2}}\left[\frac{\partial c}{\partial t}+\frac{v}{1-v c}\left(D \frac{\partial c}{\partial z}-K\right) \frac{\partial c}{\partial z}\right] \\
d x & =(1-v c) d z-v\left(D \frac{\partial c}{\partial z}-K\right) d t
\end{aligned}
$$

and

$$
t^{\prime}=t
$$

In (2.2) we have

$$
\begin{aligned}
c^{\prime} & =\frac{1}{(1-v c)}, \\
D^{\prime}\left(c^{\prime}\right) & =D\left(\frac{c^{\prime}-1}{v c^{\prime}}\right) / c^{2}
\end{aligned}
$$

and

$$
K^{\prime}\left(c^{\prime}\right)=v c^{\prime} K\left(\frac{c^{\prime}-1}{v c^{\prime}}\right),
$$

with $v$ being a translation constant. For a more detailed account of this transformation, see either [14] or [15]. The relationship between $z$ and $x$ is given by

$$
x=\int_{0}^{z}(1-v c) d \bar{z} .
$$

Applying (2.2) and (2.3a) to the boundary conditions (1.2) results in

$$
\begin{gathered}
-D^{\prime}\left(c^{\prime}\right) \frac{\partial c^{\prime}}{\partial x}+K^{\prime}\left(c^{\prime}\right)=0, \quad x=0, \\
c^{\prime} \rightarrow 1, \quad x \rightarrow \infty
\end{gathered}
$$

By introducing the new dependent variable

$$
u=c^{\prime}-1
$$

in (2.3) and (2.5), we obtain the invariant transformed problem

$$
\frac{\partial u}{\partial t}=\frac{\partial}{\partial t}\left(D^{*}(u) \frac{\partial u}{\partial x}-K^{*}(u)\right)
$$


with the boundary conditions

$$
\begin{gathered}
-D^{*}(u) \frac{\partial u}{\partial x}+K^{*}(u)=0, \quad x=0, \\
u \rightarrow 0, \quad x \rightarrow \infty,
\end{gathered}
$$

where $t^{\prime}$ has been replaced by $t$ and

$$
\begin{gathered}
D^{*}(u)=(1+u)^{-2} D\left(\frac{u}{v(1+u)}\right), \\
K^{*}(u)=v(1+u) K\left(\frac{u}{v(1+u)}\right) .
\end{gathered}
$$

The relationships between the original variables and the transformed variables are now given by

$$
\begin{aligned}
& c=\frac{u}{v(1+u)}, \\
& z=\int_{0}^{x}(1+u) d \bar{x} .
\end{aligned}
$$

From (1.3) and (2.8) the conservation condition for the transformed problem is

$$
Q^{*}=\int_{0}^{\infty} u d x
$$

where $Q^{*}=\nu Q$.

Thus, given a solution $u=g(x, t)$ to the transformed problem (2.7) and (2.9), the solution to (1.1) and (1.3) is given parametrically through

$$
\begin{aligned}
& c(x, t)=\frac{g(x, t)}{v(1+g(x, t))}, \\
& z(x, t)=x+\int_{0}^{x} g(\bar{x}, t) d \bar{x} .
\end{aligned}
$$

Therefore we can generate a new solution to $c(z, t)$ for a different class of function $D(c)$ and $K(c)$ by inverting $u(x, t)$ through (2.10). The functions $D(c)$ and $K(c)$ corresponding to the new solution are found from inverting $(2.7 \mathrm{~d})$ and $(2.7 \mathrm{e})$ as

$$
\begin{aligned}
& D(c)=\frac{1}{(1-v c)^{2}} \cdot D^{*}\left(\frac{v c}{1-v c}\right), \\
& K(c)=\frac{1}{c}(1-v c) K^{*}\left(\frac{v c}{1-v c}\right) .
\end{aligned}
$$

In Section 3, we present the known analytical solutions to $u(x, t)$ for the transformed problem where the $D^{*}(u)$ and $K^{*}(u)$ have a power law dependence on $u$. In Section 4 , we show how to invert the solution through $(2.10)$ to find $c(z, t)$, while Section 5 considers the extension to the case where $D$ and $K$ have an exponential dependence on $c$. 


\section{Similarity solution for a diffusion-convection equation}

In a recent paper [13] a similarity solution was obtained for the initial value boundary value problem

$$
\frac{\partial u}{\partial t}=\frac{\partial}{\partial x}\left(\alpha u^{m} \frac{\partial u}{\partial x}-\beta u^{m+2}\right)
$$

subject to

$$
\begin{gathered}
-\alpha u^{m} \frac{\partial u}{\partial x}+\beta u^{m+2}=0, \quad x=0, \\
u \rightarrow 0, \quad x \rightarrow \infty, \\
u(x, 0)=2 Q^{*} \delta(x),
\end{gathered}
$$

where $\alpha, \beta$ and $m$ are constants. This solution is of the form

$$
u=\left(\alpha^{-1} \beta^{2} t\right)^{-1 / m+2} f(\psi), \quad \psi=\left(\alpha^{-m-1} \beta^{m}\right)^{1 / m+2} x t^{-1 / m+2},
$$

where $f(\psi)$ is given by

$$
\begin{gathered}
\frac{d f}{d \psi}=f^{2}-\frac{1}{m+2} \psi f^{1-m}, \\
f=a, \quad \psi=0
\end{gathered}
$$

and $a$ is a constant related to the total amount $Q^{*}$ of diffusing substance. When $m=-1,0$ or $1,(3.3)$ can be integrated analytically and each of these cases is now treated separately. Since we are interested only in analytical solutions in this paper, other values of $m$ are not considered as (3.3) must then be integrated numerically.

Case 1, $m=-1$. This case was not considered by [13] although the solution to (3.3) is easily found as

$$
f(\psi)=2 a /\left(a \psi^{2}-2 a \psi+2\right), \quad \psi=x /(\beta t),
$$

and thus the solution to (3.1) is

$$
u=\alpha \beta^{-2} t^{-1} f x /(\beta t) .
$$

Case 2, $\mathbf{m}=\mathbf{0}$. This has the solution

$$
f(\psi)=\frac{a \exp \left(-\psi^{2} / 4\right)}{1-a \sqrt{\pi} \operatorname{erf}(\psi / 2)}, \quad \psi=x /(\alpha t)^{1 / 2}
$$

and

$$
u=\alpha /\left(\beta^{2} t\right)^{1 / 2} f(\psi)
$$


Case $3, \mathbf{m}=1$. For this case, the solution is

$$
\begin{aligned}
f(\psi) & =-3^{-1 / 3}\left[\frac{\sqrt{3}(S+a) A_{i}^{\prime}\left(3^{-1 / 3} \psi\right)+(S-a) B_{i}^{\prime}\left(3^{-1 / 3} \psi\right)}{\sqrt{3}(S+a) A_{i}\left(3^{-1 / 3} \psi\right)+(S-a) B_{i}\left(3^{-1 / 3} \psi\right)}\right], \\
\psi & =x\left(\beta / \alpha^{2} t\right)^{1 / 3},
\end{aligned}
$$

where $A_{i}(\psi), \beta_{i}(\psi), A_{i}^{\prime}(\psi)$ and $\beta_{i}^{\prime}(\psi)$ are the Airy functions and their derivatives, and $S=\Gamma(2 / 3) / \Gamma(1 / 3)$. The solution to (3.1) is

$$
u= \begin{cases}\left(\alpha / \beta^{2} t\right)^{1 / 3} f(\psi), & 0<\psi<\psi_{f}, \\ 0, & \psi \geq \psi_{f},\end{cases}
$$

where $\psi_{f}$, defined from the condition $f\left(\psi_{f}\right)=0$, is the smallest root of

$$
\frac{B_{i}^{\prime}\left(3^{-1 / 3} \psi\right)}{A_{i}^{\prime}\left(3^{-1 / 3} \psi\right)}+\frac{\sqrt{3}(S+a)}{S-a}=0
$$

We note that (3.9) has compact support, with the edge of the support $(X(t))$ given by $X=\psi_{f}\left(\alpha^{2} t / \beta\right)^{1 / 3}$.

\section{Results}

To construct the new solution to (1.1) and (1.2) for $c(z, t)$ and the corresponding $D(c)$ and $K(c)$, we now invert the solutions of Section 3 through (2.10) and (2.11). Care must, however, be taken for $t$ near zero if the solution is to be uniquely defined and $c(z, t)$ is to be always positive.

4.1. Forms of $D(c)$ and $K(c) \quad$ The forms of $D(c)$ and $K(c)$ are found by equating $\alpha u^{m}$ with $D^{*}(u)$ and $\beta u^{m+2}$ with $K^{*}(u)$ in (2.7). Thus, from (2.11), we have $D(c)=$ $\alpha \nu^{m} c^{m}(1-v c)^{-m-2}$ and $K(c)=\beta \nu^{m+1} c^{m+2}(1-v c)^{-m-1}$. Choosing the particular forms

$$
\begin{aligned}
& D(c)=D_{0} c^{m}(1-v c)^{-m-2}, \\
& K(c)=K_{0} c^{m+2}(1-v c)^{-m-1},
\end{aligned}
$$

where $D_{0}$ and $K_{0}$ are constants, we have

$$
\alpha=D_{0} \nu^{-m}, \quad \beta=K_{0} \nu^{-m-1},
$$

and so from (3.2)

$$
u=\frac{v f(\psi)}{\left(K_{0}^{2} t / D_{0}\right)^{1 /(m+2)}}, \quad \psi=x\left(\frac{K_{0}^{m}}{D_{0}^{m+1} t}\right)^{1 /(m+2)}
$$


While $D_{0}$ is a positive constant, $K_{0}$ may be negative or positive so that the convective term may retard or enhance the flow. In the rest of the analysis, we set $D_{0}$ to unity without loss of generality.

4.2. Behaviour of solutions for $\boldsymbol{t}$ near zero The appropriate initial condition for (1.1) with boundary condition (1.2) and $D(c)$ and $K(c)$ given by (4.1), is determined by inverting the transformations (2.8) or (2.10) on the delta function in (3.1d) and the requirement that $z=z(x, t)$ be a single-valued function of $x$ for all $t$. This uniqueness requirement, along with the positivity condition on $c$, imposes conditions on the solution $c(z, t)$ for $t$ near zero depending on whether $u(x, t)$ is positive or negative.

(i) $u \geq 0$ Substituting $u=2 Q^{*} . \delta(x)$ into (2.8) shows that $x=0$ is mapped to the interval $0 \leq z<\nu Q$ with $c=1 / \nu$ over this range. From the positivity condition on $c$, we see that $u>0$ is equivalent to $v>0$. For $x>0, z=x+v Q$ with $c=0$, the required initial distribution for $c(z, 0)$ is thus given by the step-function distribution

$$
c= \begin{cases}1 / \nu, & 0 \leq z<v Q \\ 0, & z>v Q\end{cases}
$$

The uniqueness requirement on $z(x, t)$ implies that $\partial z / \partial x>0$ or from $(2.8 \mathrm{~b})$, that $1+u \geq 0$. For $u(x, t) \geq 0$ this always holds and then (2.8a) shows $c \geq 0$.

(ii) $u<0$ When $u$ is less than zero, the uniqueness condition for $z(x, t)$ is still determined by $1+u \geq 0$ or, combining with (4.3),

$$
1+v\left(K_{0}^{2} t\right)^{-1 /(m+2)} f(\psi) \geq 0
$$

We note from (2.8a) that if $u<0$ and $1+u \geq 0$, then for $c \geq 0$ the parameter $v$ must be negative. Consequently $v<0$ is equivalent to $u<0$. Therefore, when $u$ or $v$ is less than zero, it is clear from (4.5) that the source solution will apply only for $t>t^{*}$, with $t^{*}$ given by

$$
t^{*}=K_{0}^{-2}\left(-v f_{\max }\right)^{m+2},
$$

where $f_{\max }$ is the value of $f(\psi)$ having maximum modulus in the solution domain. The required initial condition is then given by $c\left(z, t^{*}\right)$ which can be mapped back to $c\left(z^{*}, 0\right)$ by defining a new time $t-t^{*}$. 


\subsection{Solutions}

Case 1, $\mathbf{m}=-1$ From (4.1), (4.2) and (4.3), we have

$$
\begin{aligned}
D(c) & =\frac{1}{c(1-\nu c)}, \quad K(c)=K_{0} c, \\
\alpha & =\nu, \quad \beta=K_{0} \quad \text { and } \\
u & =\frac{\nu f(\psi)}{K_{0}^{2} t}, \quad \psi=\frac{x}{K_{0} t} .
\end{aligned}
$$

Thus from (2.10), (3.4) and (4.7c), the solution to (1.1) and (1.2) with $D(c)$ and $K(c)$ given by $(4.7 \mathrm{a})$ is

$$
\begin{aligned}
& c(x, t)=\frac{2 t}{x^{2}+2 t\left(\nu-K_{0} x\right)+2 a^{-1} K_{0}^{2} t^{2}} \quad \text { and } \\
& z(x, t)=x+\frac{v}{K_{0} w} \ln \left[\frac{(1-w)\left(x-K_{0} t-w K_{0} t\right)}{(1+w)\left(x-K_{0} t+w K_{0} t\right)}\right],
\end{aligned}
$$

where $w=\sqrt{1-2 a^{-1}}$.

From (2.9), we obtain the relationship between $Q$ and $a$ as

$$
Q=\frac{1}{K_{0} w} \ln \left(\frac{1-w}{1+w}\right) .
$$

The physical requirement that $0<Q<\infty$ leads to restrictions on the values that $K_{0}$ and $a$ can take. Since $w>0$, we have from (4.8) and (4.9) that $K_{0}<0$ and $a>2$. Considering the initial condition, we note first that from (3.4) and (4.7c) with $K_{0}<0$, we must have $\psi \leq 0$ and consequently (3.4) shows $f(\psi) \geq 0$ since $f^{\prime}(\psi)>0$ for $-\infty<\psi \leq 0$. The maximum value of $f(\psi)$ then occurs at $\psi=0$ and is given by $f_{\max }=a$. Thus for

(i) $v>0,(4.5)$ is satisfied for $t \geq 0$ and the initial distribution is given by (4.4);

(ii) $v<0$, (4.5) is satisfied when $t>t^{*}$, where $t^{*}=-a v / K_{0}^{2}$ and the initial distribution is given by $c\left(z, t^{*}\right)$ in (4.8).

Case 2, $m=0 \quad$ Here we have from (4.1) - (4.3) that

$$
\begin{array}{cl}
D(c)=1 /(1-v c)^{2}, & K(c)=K_{0} c^{2} /(1-v c), \\
\alpha=1, & \beta=K_{0} / \nu
\end{array}
$$

and

$$
u=\frac{\nu f(\psi)}{K_{0} t^{1 / 2}}, \quad \psi=x / t^{1 / 2}
$$


Thus from (2.10), (3.6) and (4.10c), the solution to (1.1) and (1.2), with $D(c)$ and $K(c)$ given by $(4.10 \mathrm{a})$, is

$$
\begin{gathered}
c(x, t)=\frac{\exp \left(-x^{2} /(4 t)\right)}{a^{-1} K_{0} \sqrt{t}-K_{0} \sqrt{\pi t} \operatorname{erf}(x /(2 \sqrt{t}))+v \exp \left(-x^{2} /(4 t)\right)}, \\
z(x, t)=x-\frac{v}{K_{0}} \ln [1-a \sqrt{\pi} \operatorname{erf}(x /(2 \sqrt{t}))],
\end{gathered}
$$

and

$$
Q=-\frac{1}{K_{0}} \ln [1-a \sqrt{\pi}] .
$$

From (4.12), it is evident that relevant solutions exist for the two parameter ranges

(a) $0<a<1 / \sqrt{\pi}, K_{0}>0$,

(b) $a<0, K_{0}<0$,

with the corresponding solution domain for $\psi$ being $0 \leq \psi<\infty$. For both parameter ranges (a) and (b) when $v>0$ (or $u>0$ ), the initial distribution is given by (4.4). If $v<0$, then we need to treat (a) and (b) separately as follows.

$v<0$ : When $a>0$ as in (a), then (3.3) shows $f(\psi)>0$ for $0 \leq \psi<\infty$. Hence $f_{\max }$ is the value of $f(\psi)$ at the stationary point $\psi^{*}$, where from $(3.3 \mathrm{a}), \psi^{*}$ is the root of

$$
f(\psi)-\psi / 2=0
$$

with $f(\psi)$ given by (3.6). The initial distribution is now found from (4.11) as $c\left(z, t^{*}\right)$ with $t^{*}=\left(v f_{\max } / K_{0}\right)^{2}$. When $a<0$ as in (b), then (3.3) shows that $f(\psi) \leq 0$ while $f^{\prime}(\psi) \geq 0$ for $0 \leq \psi \leq \infty$, so that $f_{\max }=a$ and $t^{*}=\left(v a / K_{0}\right)^{2}$.

Graphs of the solution $c(z, t)$ are shown in Figures 1 and 2. In Figure $1, K_{0}>0$ and the gravity convection term enhances the flow. This is seen through the maximum value for $c$ occurring at values of $z>0$ with the position of this peak increasing as $t$ increases. In Figure 2, $K_{0}<0$ and hence the gravity convection term retards the flow and the peak value of $c$ now occurs at $z=0$. We note also in Figure 2 that $\partial c / \partial z<0$ for all $z>0$ as in the case where only diffusion is controlling the flow.

Case 3, $\mathbf{m}=1 \quad$ In this case, (4.1) to (4.3) give

$$
D(c)=D_{0} c /(1-v c)^{3}, \quad K(c)=K_{0} c^{3} /(1-v c)^{2},
$$

and

$$
u=\frac{v f(\psi)}{\left(K_{0}^{2} t\right)^{1 / 3}}, \quad \psi=K_{0}^{1 / 3} x t^{-1 / 3}
$$




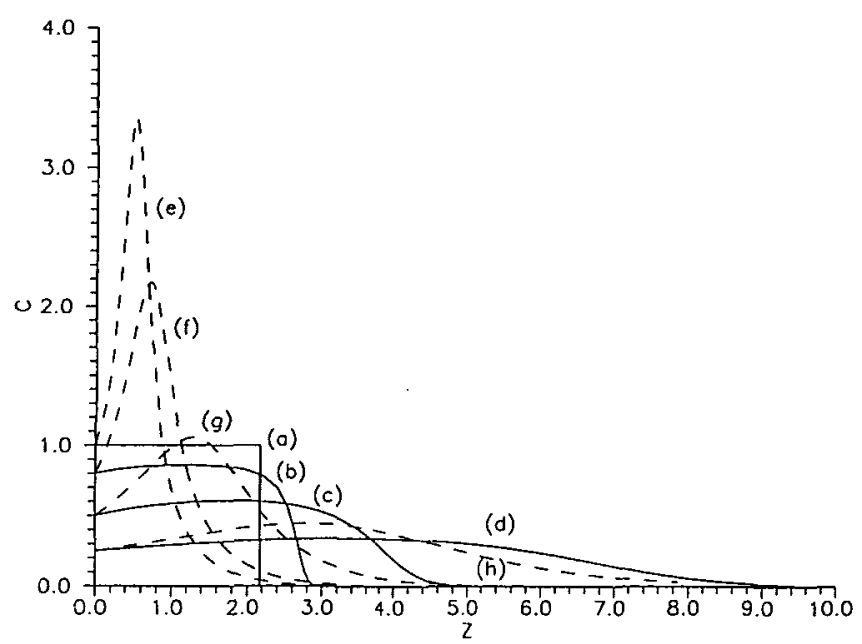

FIGURE 1. Solution (4.11) for $m=0, K_{0}=1, a=1 / 2$ and $|v|=1$. Solid lines give solutions for $\nu=1$ at times $2 t^{1 / 2}=$ (a) 0 , (b) 0.25 , (c) 1.0 and (d) 3.0. Dashed lines give solutions for $v=-1$ at times $2 t^{1 / 2}=$ (e) 2.0 , (f) 2.25 , (g) 3.0 and (h) 5.0 .

Hence $c(x, t)$ and $z(x, t)$ are now given by

$$
\begin{aligned}
& c(x, t)=\frac{f(\psi)}{\left(K_{0}^{2} t\right)^{1 / 3}+v f(\psi)}, \\
& z(x, t)=x-\frac{v}{K_{0}} \ln [w(\psi)],
\end{aligned}
$$

where

$$
w(\psi)=\frac{3^{1 / 6}}{2} \Gamma(1 / 3)\left\{\sqrt{3}(S+a) A_{i}\left(3^{-1 / 3} \psi\right)+(S-a) B_{i}\left(3^{-1 / 3} \psi\right)\right\}
$$

and $f(\psi)$ is defined by (3.8). The source strength $Q$ and the constant $a$ are related by

$$
Q=-\frac{1}{K_{0}} \ln \left[w\left(\psi_{f}\right)\right]
$$

with $\psi_{f}$ found from (3.10). Relevant solutions again exist for the two regions

(a) $K_{0}>0,0<a<S, 0 \leq \psi \leq \psi_{f}$,

(b) $K_{0}<0,0<a<\infty, \psi_{f} \leq \psi \leq 0$.

Note that for $K_{0}<0, \psi \leq 0$ and then $\psi_{f}$ is defined as the smallest negative root of (3.10). Finally, for $v>0$, the initial distribution is defined by (4.4), while for $v<0$ it is given by $c\left(z, t^{*}\right)$ with $t^{*}=K_{0}^{-2}\left(-\nu f_{\max }\right)^{3}$, where $f_{\max }=f\left(\psi^{*}\right)$ and $\psi^{*}$ is the root of

$$
f(\psi)^{2}-\psi / 3=0 .
$$

In (4.17) $f(\psi)$ is given by (3.8) and in (b) above, where $\psi \leq 0, f_{\max }=a$. Figures 3 and 4 show the solutions for $c(z, t)$ for $K_{0}>0$ and $K_{0}<0$ respectively. 


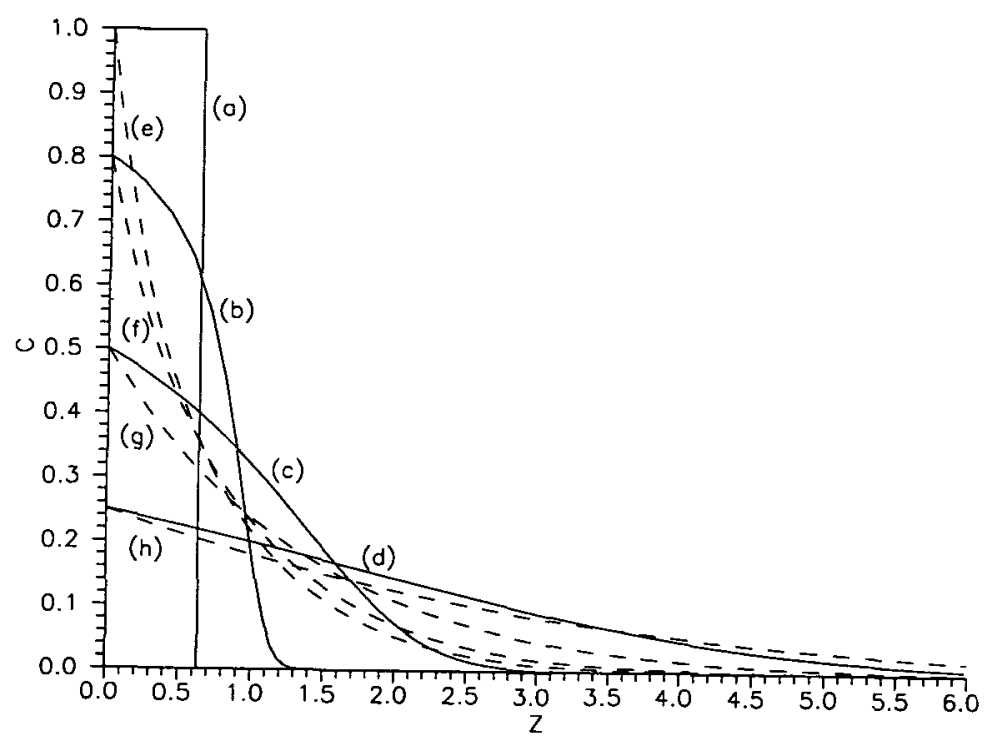

FIGURE 2. Solution (4.11) for $m=0, K_{0}=-1, a=-1 / 2$ and $|\nu|=1$. Labelling and times are the same as those used in Figure 1.

\section{Extension for exponential diffusivities and conductivities}

It is well known that the source solution for the nonlinear diffusion equation with an exponential diffusivity $D=e^{-n c}$ is the limiting solution of the power law diffusivity $D=c^{m}$ as $m \rightarrow \infty$. In this section, we seek an extension of this solution for (1.1) and (1.2). In Section 2, the variable $c$ was first translated to $1-v c$ before applying the Bäcklund transformation. Here we apply the transformation without translating $c$ or specifically ([14])

$$
\begin{gathered}
\frac{\partial c^{\prime}}{\partial x}=-\frac{1}{c^{3}} \frac{\partial c}{\partial z} \\
\frac{\partial c^{\prime}}{\partial t^{\prime}}=\frac{1}{c^{2}}\left[-\frac{\partial c}{\partial t}+\frac{1}{c}\left\{D \frac{\partial c}{\partial z}-K\right\} \frac{\partial c}{\partial z}\right], \\
d x=c d z+\left[D \frac{\partial c}{\partial z}-K\right] d t \\
t^{\prime}=t \\
c^{\prime}=1 / c, \\
z=\int_{0}^{x} c^{\prime} d \bar{x}, \quad \text { or } \quad x=\int_{0}^{z} c d \bar{z}
\end{gathered}
$$




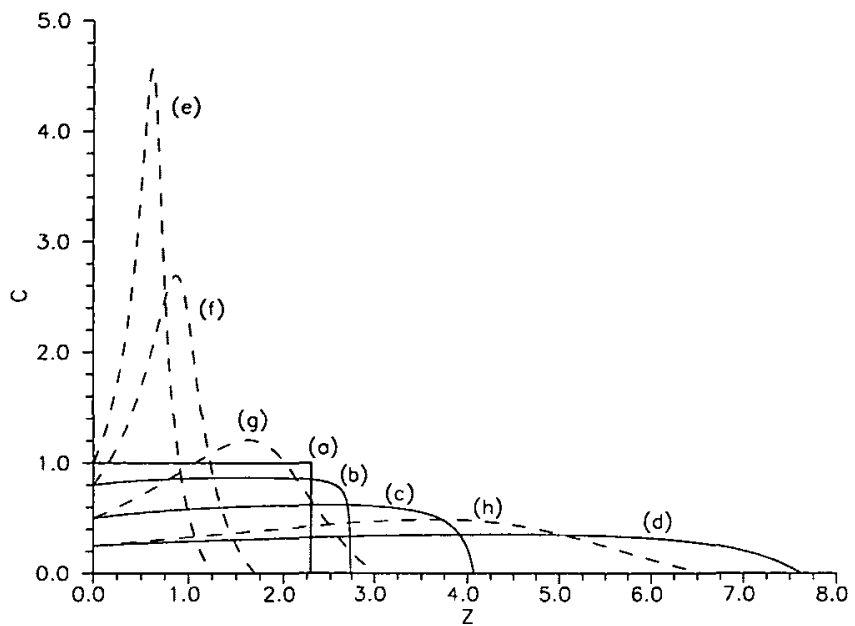

FIGURE 3. Solution (4.15) for $m=1, K_{0}=1, a=1 / 2$ and $|\nu|=1$. Solid lines give solutions for $v=1$ at times $2 t^{1 / 3}=$ (a) 0 , (b) 0.25 , (c) 1.0 and (d) 3.0. Dashed lines give solutions for $v=-1$ at times $2 t^{1 / 3}=$ (e) 2.0 , (f) 2.25 , (g) 3.0 and (h) 5.0 .

to transform (1.1) and (1.2) to

$$
\begin{gathered}
\frac{\partial c^{\prime}}{\partial t}=\frac{\partial}{\partial x}\left[D^{\prime}\left(c^{\prime}\right) \frac{\partial c^{\prime}}{\partial x}-K^{\prime}\left(c^{\prime}\right)\right], \\
-D^{\prime} \frac{\partial c^{\prime}}{\partial x}+K^{\prime}=0, \quad x=0, \\
c^{\prime} \rightarrow \infty \quad \text { as } \quad x \rightarrow Q
\end{gathered}
$$

and

$$
\begin{gathered}
D^{\prime}=c^{2} D(c), \\
K^{\prime}=-K(c) / c .
\end{gathered}
$$

In the absence of the conductivity term, we know that (5.3) has a solution for $D^{\prime}\left(c^{\prime}\right)=$ $e^{-n c^{\prime}}, n>0$ ([8]). On letting the conductivity also be an exponential of the form $K^{\prime}\left(c^{\prime}\right)=-K_{0} e^{-b c^{\prime}}$ and taking a new variable

$$
u=e^{-n c^{\prime}},
$$

(5.3) becomes

$$
\begin{gathered}
\frac{\partial u}{\partial t}=u \frac{\partial^{2} u}{\partial x^{2}}-b K_{0} u^{b / n} \frac{\partial u}{\partial x} \\
\frac{\partial u}{\partial x}-n K_{0} u^{b / n}=0, \quad x=0, \\
u=0, \quad x=Q .
\end{gathered}
$$




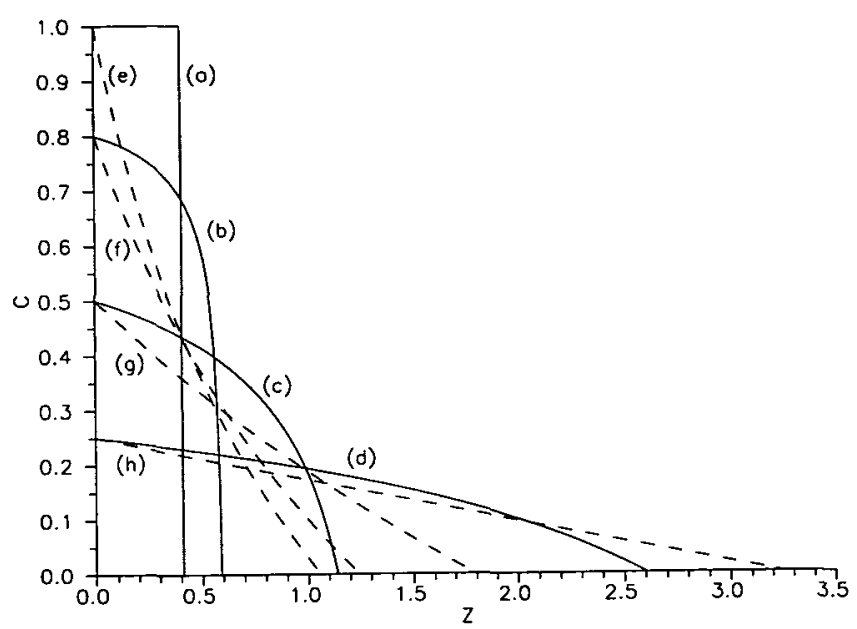

FIGURE 4. Solution (4.15) for $m=1, K_{0}=-1, a=1 / 2$ and $|\nu|=1$. Labelling and times are the same as those used in Figure 3.

It is straightforward to show that (5.6) has a separable solution in the case $b=n$ of the form $u(x, t)=f(x) \cdot g(t)$, which leads to

$$
u(x, t)=\frac{1}{\varepsilon^{2} t}\left[(1+\varepsilon x)-(1+\varepsilon Q) e^{-\varepsilon(Q-x)}\right],
$$

where $\varepsilon=n K_{0}$. Hence from (5.2), (5.4) and (5.5), the solution of (1.1) and (1.2) for

$$
\begin{gathered}
D(c)=\frac{1}{c^{2}} e^{-n / c}, \\
K(c)=K_{0} c e^{-n / c},
\end{gathered}
$$

is given by

$$
\begin{gathered}
c(x, t)=-\frac{n}{\ln [u(x, t)]}, \\
z(x, t)=-\frac{1}{n} \int_{0}^{x} \ln [u(\bar{x}, t)] d \bar{x},
\end{gathered}
$$

with $u(x, t)$ defined by (5.7).

Edwards [2] has given a similarity reduction to (5.3a) for exponential $D^{\prime}$ and $K^{\prime}$ for $b \neq n$, however it is only for $b=n$ that the resulting ordinary differential equation integrates analytically and satisfies the boundary conditions $(5.3 \mathrm{~b}, \mathrm{c})$.

It is not immediately obvious that the $D$ and $K$ of (5.8) have any physical application. For soil water flow both $D$ and $K$ are monotonically increasing functions of $c$ for $0 \leq c \leq 1$ ( $c=1$ implying a saturated soil) with $d^{2} D / d c^{2}$ and $d^{2} K / d c^{2}$ positive. For 
$n$ positive $K(c)$ satisfies these conditions automatically, however $D(c) \rightarrow 0$ as $c \rightarrow 0$ and $c \rightarrow \infty$ with the maximum occurring at $c=n / 2$. In addition $d^{2} D / d c^{2}=0$ at two values of $c$ given by $c=n(1 / 2 \pm 1 / \sqrt{12})$. Thus $D(c)$ as defined by (5.8a) has direct application to soil water flow provided $0 \leq c \leq 1$ and $n \geq 1 /(1 / 2-1 / \sqrt{12})$.

When $c$ is the soil moisture content, the capillary potential $h(h<0)$ is related to $c$ through

$$
D(c)=K(c) \frac{d h}{d c}
$$

Using $D$ and $K$ from (5.8) and integrating with the condition $c=1 h=h_{e}$ results in a soil moisture characteristic curve defined as

$$
c=\frac{1}{\left[1+2 K_{0}\left(h_{e}-h\right)\right]^{1 / 2}},
$$

where $h_{e}$ is the air entry potential. We note that (5.11) is a particular form of the van Genuchten [17] $c(h)$ model

$$
c=\frac{1}{\left[1+\lambda\left(h_{e}-h\right)^{r}\right]^{s}},
$$

where $\lambda, r$ and $s$ are fitting parameters. This model has been consistently and reliably used to model $c(h)$ curves for many soil types (Fayer and Simmons, [3]).

For (5.9) to be a valid solution, then $0 \leq u(x, t) \leq 1$, a condition which is again equivalent to a one-to-one mapping between $x$ and $z$. Therefore, from (5.7)

$$
t \geq \frac{1}{\varepsilon^{2}}\left[(1+\varepsilon x)-(1+\varepsilon Q) e^{-\varepsilon(Q-x)}\right]
$$

which requires the following two cases to be considered.

(i) $\varepsilon>0$ or $K_{0}>0$ The right-hand side of (5.13) is a concave function of $x$ downwards with its maximum occurring at

$$
x_{m}=Q-\frac{1}{\varepsilon} \ln (1+\varepsilon Q) \text {. }
$$

Thus from (5.13), our solution applies for $t \geq t^{*}$ where

$$
t^{*}=\frac{1}{\varepsilon^{2}}[\varepsilon Q-\ln (1+\varepsilon Q)]
$$

with the initial distribution $c(z)$ given by $c\left(x, t^{*}\right)$ and $z\left(x, t^{*}\right)$ in (5.9). 
(ii) $\varepsilon<0$ or $K_{0}<0 \quad$ For $0 \leq x \leq Q$, the maximum of the right-hand side of (5.13) occurs at $x_{m}=0$ so that $t^{*}$ is given by

$$
t^{*}=\frac{1}{\varepsilon^{2}}\left[1-(1+\varepsilon Q) e^{-\varepsilon Q}\right] \text {. }
$$

We note that here the special case of $Q=-1 / \varepsilon$ allows $c(z, t)$ to be found implicitly from (5.7) and (5.9) as

$$
z=\frac{1}{n}\left\{Q\left[1+\ln \left(t / Q^{2}\right)\right]-\frac{t}{Q} e^{-n / c}\left(1+\frac{n}{c}\right)\right\},
$$

with $t^{*}=Q^{2}$ from (5.16). Thus the initial profile is given by $c\left(z, Q^{2}\right)$ or

$$
z=\frac{Q}{n}\left[1-\left(1+\frac{n}{c}\right) e^{-n / c}\right] .
$$

Finally, we note that the solutions for cases (i) and (ii) above have compact support as $D(c)$ given by (5.8) is Dini continuous at $c=0$ (Peletier [11] ). For $\varepsilon \rightarrow 0$ (nonlinear diffusion only), (5.7) and (5.9) reduce to (3.3) and (3.4) of King [8], while both (5.15) and (5.16) reduce to the required $t^{*}=Q^{2} / 2$. In Figure 5, solution profiles are shown for $Q=1, \varepsilon=1$ (solid lines) and $\varepsilon=-1$ ((5.14), dashed lines) at various times. The peak values for $c$ again occur at $z>0$ when $\varepsilon>0\left(K_{0}>0\right)$ and at $z=0$ for $\varepsilon<0$.

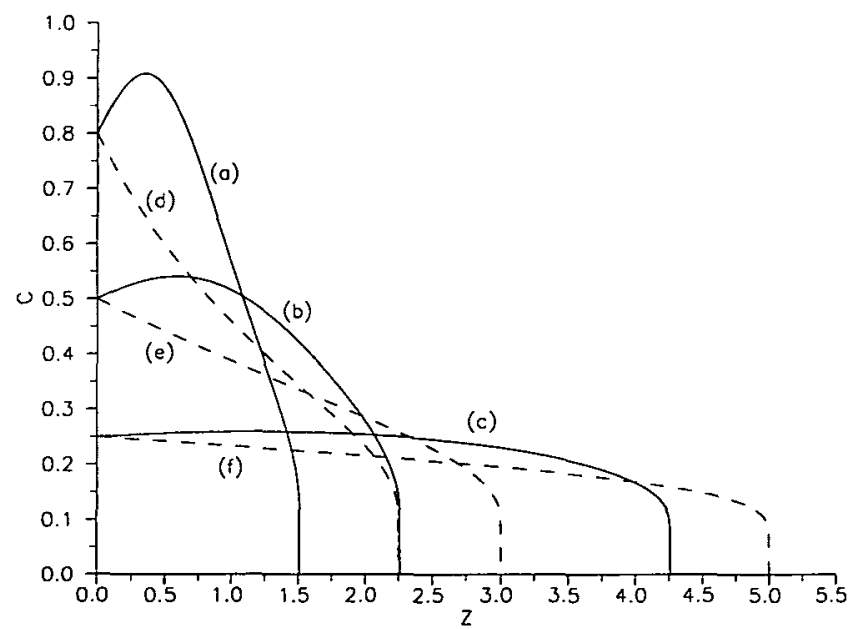

FIGURE 5. Solution curves for (5.7) and (5.9) with $Q=1, n=1$ and $|\varepsilon|=1$. Solid lines give solutions for $\varepsilon=1$ at times $-\ln \left[t^{-1}\left(1-2 e^{-1}\right)\right]=$ (a) 1.25 , (b) 2 and (c) 4 . Dashed lines give solutions for $\varepsilon=-1$ at times $\ln (t)=$ (d) 1.25 , (e) 2 and (f) 4 . 


\section{Conclusion}

In conclusion, we have presented a new range of mass-conserving source solutions to the nonlinear diffusion convection equation. These solutions extend the previous work which applied to nonlinear diffusion only. In particular, we provide solutions for the power law diffusivities and conductivities

$$
D(c)=\frac{D_{0} c^{m}}{(1-v c)^{m+2}} \quad \text { and } \quad K(c)=\frac{K_{0} c^{m+2}}{(1-v c)^{m+1}},
$$

for $m=-1,0$ and 1 , plus the exponential law diffusivity and conductivity for positive $n$

$$
D(c)=\frac{D_{0}}{c^{2}} e^{-n / c} \quad \text { and } \quad K(c)=K_{0} c e^{-n / c} .
$$

We also noted in the introduction that the flow equation under the change of variable $z=-z$ with $K_{0}>0$ is equivalent to the variable change $K_{0}=-K_{0}$ with $z>0$. Consequently the two semi-infinite domain solutions for $K_{0}>0\left(K_{0}^{+}\right)$and $K_{0}<0$ $\left(K_{0}^{-}\right)$can be joined to form a solution for the infinite domain $-\infty<z<\infty$. Denote $c^{+}(z, t)$ and $c^{-}(z, t)$ as the solutions for $K_{0}^{+}$and $K_{0}^{-}$respectively such that $K_{0}^{+}=-K_{0}^{-}$. Then if $c^{+}$and $c^{-}$also have the same values of $m, v$ and $|a|$ they can be joined at $z=0$. This is possible for the power law $D(c)$ and $K(c)$ when $m=0$ provided $0 \leq|a| \leq 1 / \sqrt{\pi}$ and when $m=1$ if $0 \leq a \leq S$ where there are solutions for $c^{+}$and $c^{-}$. No infinite domain solutions exist for $m=-1$, since $c^{+}$does not exist. For exponential $D(c)$ and $K(c)$ we require (apart from $K_{0}^{+}=-K_{0}^{-}$) that $c^{+}$and $c^{-}$ have the same $n$ and $Q$ only. The source strength for the infinite domain solution is simply the sum of the individual source strengths for $c^{+}$and $c^{-}$.

\section{References}

[1] P. Broadbridge and C. Rogers, "Exact solutions for vertical drainage and redistribution in soils", J. Eng. Math. 24 (1990) 25-43.

[2] M. P. Edwards, "Classical symmetry reductions of nonlinear diffusion - convection equations", Phys. Lett. 190 (1994) 149-154.

[3] M. J. Fayer and C. S. Simmons, "Modified soil water retention functions for all matric suctions", Water Resour. Res. 31 (1995) 1233-1238.

[4] H. Fujita, "The exact pattern of a concentration - dependent diffusion in a semi - infinite medium, II", Textile Res. J. 22 (1952) 823-827.

[5] R. E. Grundy, "The Cauchy problem for a nonlinear diffusion equation with absorption and convection", IMA J. Appl. Math. 40 (1988) 183-204.

[6] J. M. Hill, "Similarity solutions for nonlinear diffusion in a new integration procedure", J. Eng. Math. 23 (1989) 141-155.

[7] W. L Hogarth, J.-Y. Parlange and R. D. Braddock, "First integrals of the infiltration equation. 2. Nonlinear conductivity", Soil Sci. 148 (1989) 165-171. 
[8] J. R. King, "Exact solutions to some nonlinear diffusion equations", Q. J. Mech. Appl. Math. 42 (1989) 537-552.

[9] J. G. Kingston and C. Rogers, "Reciprocal Bäcklund transformations of conservation laws", Phys. Lett. 92 (1982) 261-264.

[10] I. G. Lisle and J.-Y. Parlange, "Analytical reduction for a concentration dependent diffusion problem", ZAMP 44 (1993) 85-102.

[11] L. A. Peletier, "A necessary and sufficient condition for the existence of an interface in flows through porous media", Arch. Rat. Mech. Anal. 56 (1974) 183-190.

[12] J. R. Philip, "Theory of infiltration", Adv. Hydro 5 (1969) 215-296.

[13] J. R. Philip, "Exact solutions for redistribution by nonlinear convection-diffusion", J. Aust. Math. Soc. Ser. B 33 (1992) 363-383.

[14] C. Rogers, "Application of reciprocal Bäcklund transformations to a class of nonlinear boundary value problems", J. Phys. A: Math. Gen. 16 (1983) L493-L495.

[15] G. C. Sander, "Exact solutions to nonlinear diffusion-convection problems on finite domains", $J$. Aust. Math. Soc. Ser. B 33 (1991) 384-401.

[16] G. C. Sander, I. F. Cunning, W. L. Hogarth and J.-Y. Parlange, "Exact solution for nonlinear, nonhysteretic redistribution in vertical soil of finite depth", Water Resour. Res. 27 (1991) 15291536.

[17] R. van Genuchten, "Predicting the hydraulic conductivity of unsaturated soils", Soil Sci. Soc. Am. J. 44 (1980) 892-898.

[18] A. W. Warrick, D. O. Lomen and A. Islas, "An anlytical solution to Richards' equation for a draining soil profile”, Water Resour. Res. 26 (1990) 253-258.

[19] K. K. Watson, R. J. Reginato and R. D. Jackson, "Soil water hysteresis in a field soil", Soil Sci. Soc. Am. Proc. 39 (1975) 242-246.

[20] K. K. Watson and V. Sardana, "Numerical study of the effect of hysteresis on post infiltration redistribution", in Interm. Conf. on Infiltration Development and Application, Univ of Hawaii, Jan $6-7,1987)$.

[21] C. M. Yung, K. Verburg and P. Baveye, "Group classification and symmetry reductions of the nonlinear diffusion - convection equation $u_{t}=\left(d(u) u_{x}\right)_{x}-k^{\prime}(u) u_{x}$, Int. J. Non-Linear Mech. 29 (1994) 273-278. 